\title{
Challenges in Tuberculosis Diagnosis and Management: Recommendations of the Expert Panel
}

uberculosis (TB), known with several other names such as Tapedik, Kshay Rog, phthisis, consumption, white death, great white plague, graveyard cough, King's Evil, etc., is documented since 3000 years BC and some workers rightly consider it a disease of antiquity. However, the pathogen causing this disease (Mycobacterium tuberculosis) was discovered and named 133 years back on this day by Dr. Robert Koch. Even in modern times, during the World War I and II, TB was a major handicap for US and European armies, killing 1000's of them. However, with the improvement in the socioeconomic standards coupled with social etiquettes (no spitting in public places) the incidence of TB reduced dramatically in western world and TB became a disease of poor countries with poor hygienic conditions.

The disease remains a major health problem in these countries. The data of year 2013 published by WHO in its 2014 report, showed that globally 9 million persons developed TB and 1.5 million die every year, most of them were HIV negative..$^{[1]}$ This figure is higher than 2012 data, indicating that TB prevalence is not declining on the expected lines. More worry-some issue is fast spread of multidrug resistant (MDR) TB, which is estimated to be in more than 4,80,000 patients globally and almost half of them are dying. The BRICS (India China, South Africa, Russia and Brazil) countries remain high TB burden regions, but most cases are harbored in India and China only due to high population. In these two countries, more than half of the TB cases still remain undetected and continue to spread TB in the community unknowingly. India alone accounts for more than 2 million new cases and several 1000's die every year. ${ }^{[2]} \mathrm{It}$ is probably due to lack of awareness about the disease and the facilities made available to the public by Government for early diagnosis and treatment.

\begin{tabular}{|l|l|}
\hline \multicolumn{3}{c}{ Access this article online } \\
\hline Quick Response Code: & Website: \\
\hline & www.jponline.org \\
\hline & \\
\hline
\end{tabular}

The WHO and stop-TB partner publications give a mixed feeling. While millions of Pakistani children are still left unvaccinated due to short supply of BCG vaccine, and almost half of the TB patients are given inappropriate drugs (drugs to which the pathogen is not susceptible) in Bombay; but on a good note, rates of some important drugs like cycloserine have been reduced up to $55 \%$, making several low-income countries to afford it. Furthermore, several drugs have been re-purposed for TB treatment such as carbapenems, and fluoroquinolones. A new drug Bedaquiline, though still in clinical trial stage, has raised hopes in patients infected with totally drug resistant or extremely drug-resistant $M$. tuberculosis.

However, the biggest challenge is early and species specific diagnosis of TB, detection of its drug resistance pattern and availability of highly active drug treatment, which is short course, preferably a few weeks. For prevention, the most desirable approach, which one can dream is potent preventive vaccine. Unfortunately, we are not close to any of these. Even though GeneXpert assay has changed the diagnostic scenario in last few years, due to its ability to diagnose a case of $\mathrm{TB}$ within $2 \mathrm{~h}$ and innovative sample processing protocol. After its endorsement by WHO, more than 3550 GeneXpert machines have been installed globally (mostly in Africa) including more than 300 machines in India. In Africa where this system is system is being used for more than 3 years as first line test, the recent data indicates that roll out of GeneXpert has not made desirable impact on the TB control programs in these countries. Another pertinent issue is about unaffordable cost of system, its cartridges and annual maintenance, especially after the international donations are stopped. In remote areas, where temperature and electricity are the concerns, a recent publication from Dhule, Maharashtra, shows that about $48 \%$ suspected MDR cases were GeneXpert negative but smear positive. ${ }^{[3]}$ This needs to be examined, if these were due to non-tuberculous mycobacteria or GeneXpert gave actually false negative results. Another issue is that rifampin resistance is considered as surrogate marker of MDR, thus ignoring the importance of $\mathrm{INH}$, which itself is a potential anti-TB drug. Several 
other molecular tests are also in pipeline, or in evaluation stage and some are already endorsed, such as line probe assay. However, molecular tests will ever be taken as first line of test for drug susceptibility profiling is a big question, mainly because several mutations occur outside RRDR and other unknown mechanisms are involved in drug resistance, which cannot be addressed by these molecular tests. Hence, we still have to rely upon culture based phenotypic detection methods as standard methods albeit at the cost of longer turnaround time. The Government of India has prioritized drug resistant $\mathrm{TB}$, co-morbidities, partnership with private sector, pediatric TB and urban TB in its new strategic plan (2012-2017)..$^{[2]}$

To discuss various challenges in diagnosis and management of TB a day long brainstorming symposium was held at the All India Institute of Medical Sciences, New Delhi on $24^{\text {th }}$ March 2015, on the occasion of World TB day. The symposium was followed by panel discussions. The recommendations of the panel were clear and emphasized that in the field of TB diagnosis and management, there are several challenges which have not yet attracted the attention of program managers and the funding agencies. Thus these challenges also need to be addressed on top priority. These challenges include nonavailability of point-of-care (POC) test which can reach to the remotest area. Furthermore, even though there are several new initiatives taken up by the Government and other partners these newer technologies and information are not reaching to these areas. The experts were also of the unanimous opinion that diagnosis of extra-pulmonary tuberculosis (EPTB) is a major challenge. It is for two reasons, the first is due to the difficulty in obtaining appropriate clinical sample, especially from the nonreachable sites and secondly due to the poor sensitivity of diagnostic tests. An example of GeneXpert was given which has very poor detection rate of $\mathrm{TB}$ in ascitic and pleural fluids. ${ }^{[4]}$ Though liquid culture has reasonably acceptable detection rate, but it takes 2-3 weeks to grow the mycobacteria. Therefore, it was proposed that there is an urgent need to develop biomarkers for the diagnosis of EPTB, preferably using alternative samples such as whole blood, serum/plasma, urine, sweat etc., The issue of nonavailability of markers and methods for early diagnosis, prognosis and outcome (cure or treatment failure) was also considered a priority research area. It was also discussed and agreed upon that there is urgent need for finding out host/mycobacterial biomarkers (cell based or serum based), which can predict the development of disease from latent TB. There is limited data on alternative clinical samples for PTB as well as for EPTB (e.g., urine, blood, saliva, buccal swabs, etc.) specially in HIV-TB co-infected patients, and thus this area needs urgent attention of funding agencies. It will also be highly desirable to have Biomarkers of treatment compliance/noncompliance of treatment and reasons where the in vitro - drug susceptibility reports indicates susceptible bacteria but clinically there is no response. Outcome of this research can directly help the national TB control programs. The experts were also concerned about the potency of anti-TB drugs, which are questioned by some activists time to time. It was also felt very pertinent that if the dosage and duration of treatment should be modified according to the mycobacterial burden on the host (AFB smear grading). It would also be highly desirable to develop Novel and highly specific test(s) to rule out $\mathrm{TB}$ or a highly sensitive screening test, commonly known as triage test.

The ideal triage test could be a biomarker in a POC format, or handheld X-ray or adhesive tapes. In a recent publication, Singh et al. found four novel antigens, which are highly sensitive as well as specific for diagnosing and detecting MDR-TB. These antigens have been tested at laboratory scale with high sensitive and specificity. ${ }^{[5]}$ The field trials will be undertaken soon. In the next few months, they shall be evaluating the utility of an adhesive sensor (developed by a group of Israeli Scientists), the sensor will be taped on skin of chest or arm and after $24 \mathrm{~h}$, if the patient is having TB the sensor will turn red and if the person is not having TB the sensor will turn green. The volatile organic compounds absorbed onto the tape will be further analyzed using nano-particle sensor technology. Thus, these can be used as highly successful triage tests.

\section{Sarman Singh, Bindu Dey ${ }^{1}$, KS Sachdeva ${ }^{2}$, SK Kabra ${ }^{3}$, KK Chopra ${ }^{4}$, Vijay K Chaudhary ${ }^{5}$, Pawan Sharma', VM Katoch}

\author{
Department of Laboratory Medicine, Division of \\ Clinical Microbiology and Molecular Medicine, \\ ${ }^{3}$ Department of Paediatrics, Paediatric Pulmonary \\ Division, All India Institute of Medical Sciences, \\ 'Department of Biotechnology, Government of India, \\ ${ }^{2}$ Central TB Division, Directorate General of Health Services, \\ Government of India, ${ }^{4}$ New Delhi Tuberculosis Center, \\ ${ }^{5}$ Department of Biochemistry, University of Delhi-South \\ Campus, ${ }^{6}$ Department of Health Research and Indian Council \\ of Medical Research, Government of India, New Delhi, India
}

Address for correspondence: Prof. Sarman Singh, E-mail: Sarman_singh@yahoo.com 


\section{REFERENCES}

1. World Health Organization. Global Tuberculosis Report 2014. Geneva, Switzerland: WHO; 2014. Available from: http://www.who.int/tb/ publications/global_report/en/. [Last accessed on 2015 Apr 02].

2. Tuberculosis Control - India. Available from: http://www.tbcindia.nic. in/. [Last accessed on 2015 Mar 31].

3. Uday SS, Chandrashekar DS, Kashinath KR, Nandkumar DM. GeneXpert MTB/RIF for rapid identification of clinically suspected MDR-TB cases. J Med Sci Clin Res 2015;3:4661-8.

4. Penz E, Boffa J, Roberts DJ, Fisher D, Cooper R, Ronksley PE, et al. Diagnostic accuracy of the Xpert ${ }^{\circledR}$ MTB/RIF assay for extra-pulmonary tuberculosis: A meta-analysis. Int J Tuberc Lung Dis 2015;19:278-84, i-iii.

5. Singh A, Gopinath K, Sharma P, Bisht D, Sharma P, Singh N, et al. Comparative proteomic analysis of sequential isolates of Mycobacterium tuberculosis from a patient with pulmonary tuberculosis turning from drug sensitive to multidrug resistant. Indian J Med Res 2015;141:27-45.

How to cite this article: Singh S, Dey B, Sachdeva KS, Kabra SK, Chopra KK, Chaudhary VK, et al. Challenges in tuberculosis diagnosis and management: Recommendations of the expert panel. J Lab Physicians $2015 ; 7: 1-3$

\section{"Quick Response Code" link for full text articles}

The journal issue has a unique new feature for reaching to the journal's website without typing a single letter. Each article on its first page has a "Quick Response Code". Using any mobile or other hand-held device with camera and GPRS/other internet source, one can reach to the full text of that particular article on the journal's website. Start a QR-code reading software (see list of free applications from http://tinyurl.com/ yzlh2tc) and point the camera to the QR-code printed in the journal. It will automatically take you to the HTML full text of that article. One can also use a desktop or laptop with web camera for similar functionality. See http://tinyurl.com/2bw7fn3 or http://tinyurl.com/3ysr3me for the free applications. 